\title{
Two Variational Problems in Hermitian Geometry
}

\author{
ISAAC CHAVEL
}

\author{
Communicated by S. S. CHerN
}

Let $M$ be a compact connected $2 n$-dimensional complex analytic Hermitian manifold ( $n$ is the complex dimension), with associated 2-form $\Omega$. ( $\Omega$ uniquely determines the metric and we often refer to the metric as $\Omega$. Also $\Omega^{n}$ is $n$ ! times the volume element of the induced Riemannian metric.) We let $R$ denote the Hermitian scalar curvature, and $\Delta$ the Hermitian-Laplace operator of the Hermitian metric ( $c f$. definitions below). Then the integral

$$
I(\Omega)=\int_{M} R^{n} \Omega^{n}
$$

is an invariant of the metric in the sense that if the metric is multiplied by a constant, the integral remains the same. In this note we prove

Theorem 1. A Hermitian metric $\Omega_{0}$ is a critical point of the functional $I(\Omega)$ where $\Omega$ varies over all Hermitian metrics - if and only if either the Hermitian scalar curvature of $\Omega_{0}$ vanishes identically on $M$, or $\Omega_{0}$ is a Hermitian-Einstein metric. In the Hermitian-Einstein case, $\Omega_{0}$ is Kählerian, i.e. $d \Omega_{0}=0$ (d denotes exterior differentiation) if and only if $\Delta_{0}$ is self-adjoint elliptic.

The Riemannian version of this theorem has been investigated originally by A. Einstein [8] and D. Hilbert [9], and more recently by M. Berger [3], and T. Nagano [11]. Indeed, one has that a Riemannian metric is Einstein if and only if its first variation of total scalar curvature vanishes.

In $\S 1$. we define the necessary concepts, and in $\$ 2$ calculate the first variation of total scalar curvature, and prove Theorem 1 . In $\$ 3$ we restrict our attention to conformal deformations of a fixed Hermitian metric and prove

Theorem 2. Assume $n>1$, and let $\Omega_{0}$ be a Hermitian metric such that $\Delta_{0}$ is self-adjoint, elliptic. Then $\Omega_{0}$ is a critical point of $I(\Omega)$-where $\Omega$ varies over Hermi-

* Partially supported by National Science Foundation Grant No. GP-8691. 
tian metrics of the form $\Omega=e^{2 u} \Omega_{0}$, where $u: M \rightarrow \mathrm{R}^{+}-\{0\}$ is a $C^{\infty}$ function-if and only if $\Omega_{0}$ has constant Hermitian scalar curvature $R_{0}$. In such a case, the second variation of $I(\Omega)$ is non-negative and is zero if and only if $R_{0} \equiv 0$, or the infinitesmal deformation is a constant.

Note that for $n=1$, Theorem 2 is false by the Gauss-Bonnet Theorem.

In the second part of this paper we consider the following: Let $C P_{n}$ be $n$-(complex) dimensional complex projective space and $C P_{k}$ a naturally imbedded subspace, $k=1, \cdots, n-1$. For any Hermitian metric $\Phi$ on $C P_{n}$ consider the number

$$
\lambda(\Phi)=\frac{\inf _{M} \int_{M} \Phi^{k}}{\left\{\int_{C P_{n}} \Phi^{n}\right\}^{k / n}} .
$$

where $M$ ranges over all compact real submanifolds homologous to $C P_{k}$. In $\$ 4$ we prove

Theorem 3. For any Kählerian metric $\Phi, \lambda(\Phi)=1,[2$, p. 311]. Also, any Kählerian metric is a local maximum of the function $\lambda$ (of Hermitian metrics), and for any deformation of a Kählerian metric, the first variation of $\lambda$ vanishes if and only if the infinitesmal deformation is Kählerian.

This theorem is a weak generalization of the calculation of the extremal length of the family of curves in a given homology class of a Riemann surface. We refer the reader to $[1 ; 2$, pp. 303-311; 4; 12] for details of that theory. We also note that by Wirtinger's Inequality [13], replacing the numerator by $k$ dimensional volume (induced by the associated Riemannian metric) increases the integral, unless $M$ is a complex submanifold. We finally note ( $c f . \S 4$ ) that if the Kählerian metrics do not realize $\sup _{\Phi} \lambda(\Phi)$ then no Hermitian metric does.

\$1. Review of formulae of Hermitian geometry. We calculate with the method of moving frames. For the necessary background the reader is referred to $[6 ; 7]$.

Let $($,$) denote the Hermitian inner product, and let e_{1}, \cdots, e_{n}$ be a local unitary frame on $M$, i.e., $\left(e_{j}, e_{k}\right)=\delta_{i k}\left(\delta_{j k}\right.$ is the Kronecker delta. All indices range from 1 to $n$ ). Also we let $\omega_{1}, \cdots, \omega_{n}$ be the co-frame of $(1,0)$-forms dual to $e_{1}, \cdots, e_{n}$. Then the Hermitian inner product is locally given by

$$
d s^{2}=\Sigma_{k} \omega_{k} \bar{\omega}_{k}
$$

and the associated 2-form by

$$
\Omega=\frac{(-1)^{1 / 2}}{2} \Sigma_{k} \omega_{k} \wedge \bar{\omega}_{k}
$$

The Hermitian connection is given locally by a unique skew-Hermitian matrix $\left(\omega_{j k}\right)$ of local 1-forms such that the local forms 


$$
\Omega_{i}=d \omega_{i}-\Sigma_{k} \omega_{k} \wedge \omega_{k i},
$$

are of type $(2,0)$. One easily checks that the forms $\Omega_{i}$ vanish identically if and only if $d \Omega=0$, i.e., $\Omega$ is Kählerian. The curvature matrix $\left(\Omega_{j_{k}}\right)$ is given by

$$
\Omega_{j k}=d \omega_{i k}-\Sigma_{l} \omega_{i l} \wedge \omega_{l k} .
$$

Each of the forms is of type $(1,1)$ and the matrix $\left(\Omega_{i k}\right)$ is skew-Hermitian and tensorial of adjoint type. $c f .\left[6\right.$, p. 36]. Therefore, if we let $\omega=\Sigma_{k} \omega_{k k}$ then $\rho$ dof $d \omega$ is invariant under change of frames and hence defines a global closed 2 -form of type $(1,1)$ on $M$, which is given locally (using (5)) by

$$
\rho=\Sigma_{k} \Omega_{k \boldsymbol{k}}
$$

$\rho$ is called the Ricci form, is pure imaginary, i.e., $\bar{\rho}=-\rho$, and is of the form

$$
\rho=\frac{1}{2} \Sigma_{i, k} R_{j k} \omega_{i} \wedge \bar{\omega}_{k},
$$

where $\bar{R}_{i k}=R_{k i}$. We denote the matrix $\left(R_{i k}\right)$ by $\Omega$ and let

$$
R=\operatorname{tr} R=\Sigma_{k} R_{k k},
$$

be the Hermitian-scalar curvature. It is a real-valued function on $\boldsymbol{M}$.

We now let $u: M \rightarrow \mathrm{R}$ be $C^{\infty}$. Then we can write $d u=\Sigma_{k}\left(u_{k} \omega_{k}+\bar{u}_{k} \bar{\omega}_{k}\right)=$ $\partial u+\bar{\partial} u$, where $\partial u=\Sigma_{k} u_{k} \omega_{k}$. Then using (4), one has

$$
\begin{aligned}
\bar{\partial} \partial u & =d(\partial u) \\
& =\Sigma_{k}\left(\left(d u_{k}-\Sigma_{l} u_{l} \omega_{k l}\right) \wedge \omega_{k}+u_{k} \Omega_{k}\right) .
\end{aligned}
$$

If we set

$$
d u_{k}-\Sigma_{l} u_{l} \omega_{k l}=\Sigma_{s} u_{k s}^{\prime} \omega_{s}+u_{k s}^{\prime \prime} \bar{\omega}_{s}
$$

then we have

$$
\bar{\partial} \partial u=-\Sigma_{k, s} u_{k s}^{\prime \prime} \omega_{k} \wedge \bar{\omega}_{s},
$$

and $u_{k s}^{\prime \prime}=\bar{u}_{s k}^{\prime \prime}$. Following S. S. Chern [7], except for a minus sign, we define

$$
\Delta u=-4 \Sigma_{k} u_{k k}^{\prime \prime} \text {. }
$$

To relate $\Delta u$ to the Laplace-Beltrami operator (which we denote by) $\square u$, we first note that if the forms $\Omega_{i}, j=1, \cdots, n$ are written as

$$
\Omega_{k}=\frac{1}{2} \Sigma_{r, s} T_{k r s} \omega_{r} \wedge \omega_{s} \quad T_{k r s}+T_{k s r}=0,
$$

then

$$
\varphi=\Sigma_{k, l} T_{k k l} \omega_{l}
$$

defines a global differential form on $M$. Let * denote the Hodge isomorphism, $\delta=-^{*} d^{*}$ the adjoint of $d, \square=d \delta+\delta d$, then $(c f .[5$, p. 530]) for a real valued function $u: M \rightarrow \mathrm{R}$,

$$
\square u=\Delta u+[d u, \varphi+\bar{\varphi}],
$$


where [ , ] is the inner product of covectors at any given point. In particular $\square=\Delta$ if and only if $\varphi=0$.

§2. The first variation of $\mathrm{I}(\Omega)$. We now fix $\Omega=\left((-1)^{1 / 2} / 2\right) \Sigma_{k} \omega_{k} \wedge \omega_{k}$ and set

$$
\Omega_{t}=\Omega+t \xi
$$

where

$$
\left\{\begin{aligned}
\xi & =\frac{(-1)^{1 / 2}}{2} \Sigma_{i, k} h_{j k} \omega_{i} \wedge \bar{\omega}_{k} \\
h_{i k} & =\bar{h}_{k i}
\end{aligned}\right.
$$

Let $\mathfrak{F}$ denote the matrix $\left(h_{i k}\right)$; then $h=\operatorname{tr} \mathfrak{F C}=\Sigma_{k} h_{k k}$ is a real-valued function on $M$. For each $t$, let $\rho_{t}, R_{t}$ denote the Ricci form and Hermitian-scalar curvature, respectively, corresponding to the Hermitian metric $\Omega_{t}$. Then by [10, p. 158] we have

$$
\begin{aligned}
\rho_{t} & =\rho+t \bar{\partial} \partial h+t^{2}(\cdots), \\
R_{t} & =R+t\left(\frac{1}{2} \Delta h-\operatorname{tr}(\text { (RC) })+t^{2}(\cdots)\right.
\end{aligned}
$$

from which one obtains

$$
R_{t}^{n} \Omega_{t}^{n}=\left(R^{n}+\operatorname{tr}\left(\frac{1}{2} R^{n-1} \Delta h-R^{n-1} \operatorname{tr}(\mathcal{B S C})+R^{n} h / n\right)+t^{2}(\cdots)\right) \Omega^{n} .
$$

Therefore if we set $I(t)=I\left(\Omega_{t}\right)$ then using $(6)$ we have

$$
\begin{aligned}
I^{\prime}(0) & =n \int_{M} R^{n-1}\left(\frac{1}{2} \Delta h+\frac{1}{n} h R-\operatorname{tr}(\mathbb{R} \mathcal{C})\right) \Omega^{n} \\
& =n \int_{M}\left\langle\langle(\mathcal{B}, \mathfrak{H C}\rangle\rangle \Omega^{n},\right.
\end{aligned}
$$

where

$$
\begin{gathered}
\Re=\left(\frac{1}{2} \square R^{n-1}+\frac{1}{2} \delta\left(R^{n-1}(\varphi+\bar{\varphi})\right)+\frac{R^{n}}{n}\right) I-R^{n-1} \Re \\
\langle\langle\circlearrowleft, \mathcal{F}\rangle\rangle=\operatorname{tr} \circlearrowleft \mathcal{F C}
\end{gathered}
$$

where $\mathcal{B}, \mathfrak{F}$ are Hermitian matrices. Therefore, $I^{\prime}(0)=0$ for all $\xi$, i.e., for all $\mathfrak{F C}$, if and only if either $R \equiv 0$, or (since tr is positive definite on Hermitian matrices) $\Theta \equiv 0$, i.e., $\Omega$ is Hermitian-Einstein. In particular, $\rho=-2(-)^{1 / 2}(R / n) \Omega$.

We now turn to the last statement of Theorem 1 . If $\Omega$ is Kählerian, then $\Omega_{i}=0, j=1, \cdots, n$, which implies $\varphi=0$ and $\square=\Delta$. Conversely, if $\Delta$ is self-adjoint elliptic then (8) can be written as

$$
\Theta=\left(\frac{1}{2} \Delta R^{n-1}+R^{n} / n\right) I-R^{n-1} \text { R. }
$$

Then $\Delta R=0, R \equiv$ const, and $\rho=-2(-1)^{1 / 2}(R / n) \Omega$ implies $d \Omega=0$. 
\$3. Conformal deformations of Hermitian metrics. As before, assume the original metric is given by

$$
d s^{2}=\Sigma_{k} \omega_{k} \bar{\omega}_{k}, \Omega=\frac{(-1)^{1 / 2}}{2} \Sigma_{k} \omega_{k} \wedge \bar{\omega}_{k}
$$

and the new metric given by

$$
d r^{2}=\Sigma_{k} \varphi_{k} \bar{\varphi}_{k}, \Phi=\frac{(-1)^{1 / 2}}{2} \Sigma_{k} \varphi_{k} \wedge \bar{\varphi}_{k}
$$

where

$$
\varphi_{k}=e^{u} \omega_{k}
$$

Then the first structure equation is given by

$$
d \varphi_{i}=\Sigma_{k} \varphi_{k} \wedge \varphi_{k j}+\Phi_{i}
$$

where

$$
\begin{aligned}
\varphi_{k i} & =(\partial-\bar{\partial}) u \cdot \delta_{k i}+\omega_{k i}, \\
\Phi_{i} & =2 \partial u \wedge \varphi_{i}+e^{u} \Omega_{i} .
\end{aligned}
$$

(In particular, if $n>1$, and the original metric is Kählerian, then the new metric is Kählerian if and only if $u \equiv$ const.) If $\left(\Phi_{i k}\right)$ is the curvature matrix of the new connection then

$$
\Phi_{i k}=\Omega_{j k}+2 \bar{\partial} \partial u \cdot \delta_{j k},
$$

from which the Ricci form $\zeta$ of the new metric is seen to be

$$
\zeta=\rho+2 n \bar{\partial} \partial u,
$$

and the scalar curvature, $S$, is given by

$$
S e^{2 u}=R+n \Delta u \text {. }
$$

We therefore have

$$
S \Phi=(R+n \Delta u) \Omega .
$$

Now let $\mu: M \times\left(-\frac{1}{2}, \frac{1}{2}\right) \rightarrow \mathrm{R}$ be given by

$$
\mu(x, t)=t u_{1}(x)+t^{2} u_{2}(x)+t^{3}(\cdots),
$$

and $\mu_{t}: M \rightarrow \mathrm{R}$ be given by $\mu_{t}(x)=\mu(x, t)$. For each $t \varepsilon\left(-\frac{1}{2}, \frac{1}{2}\right)$, let $S_{t}, \Phi_{\mathrm{t}}$ denote the scalar curvature, and associated 2-form of the Hermitian metric corresponding to the function $\mu_{t}$, as described above. Then

$$
\begin{aligned}
\left(S_{t} \Phi_{t}\right)^{n} & =\left(R+n \Delta \mu_{t}\right)^{n} \Omega^{n} \\
& =\left(R^{n}+t \alpha_{1}+t^{2} \alpha_{2}+t^{3}(\cdots)\right) \Omega^{n},
\end{aligned}
$$


where

$$
\begin{aligned}
& \alpha_{1}=n^{2} R^{n-1} \Delta u_{1}, \\
& \alpha_{2}=n^{2} R^{n-1}\left(n(n-1) \Delta u_{2}+R\left(\Delta u_{1}\right)^{2}\right) .
\end{aligned}
$$

Therefore if $I(t)=\int_{M} S_{t}^{n} \Phi_{t}^{n}$ then $I^{\prime}(0)=0$ for all $u_{1}$ if and only if

$$
\int_{M} R^{n-1} \Delta u_{1} \Omega^{n}=0
$$

for all $u_{1} . \Delta$ is self-adjoint elliptic then implies $R \equiv$ const. In such a case, for any $u_{1}, u_{2}$

$$
\begin{aligned}
I^{\prime \prime}(0) & =n^{2} R^{n-1} \int_{M}\left(n(n-1) \Delta u_{2}+R\left(\Delta u_{1}\right)^{2}\right) \Omega^{n} \\
& =n^{2} R^{n} \int_{M}\left(\Delta u_{1}\right)^{2} \Omega^{n} \\
& \geqq 0,
\end{aligned}
$$

which is equal to zero if and only if $R \equiv 0$ or $u_{1} \equiv$ const.

\$4. Proof of Theorem 3. For the rest of the paper we will let $\Omega$ be the canonical 2-form associated with the Fubini-Study metric on $C P_{n}$, normalized so that

$$
\int_{C P_{k}} \Omega^{k}=1,
$$

for all naturally imbedded $C P_{k} \subseteq C P_{n}, k=1, \cdots, n$. Now $d \Omega=0$, and for any other Kähler metric, $\Phi$, we have $\Phi=\kappa \Omega+d \alpha$, where $\kappa$ is a constant and $\alpha$ is a real 1-form. Since the exterior product of a closed form and an exact form is exact, we have

$$
\begin{aligned}
\frac{\int_{M} \Phi^{k}}{\left\{\int_{C P_{n}} \Phi^{n}\right\}^{k / n}} & =\frac{\int_{M} \kappa^{k} \Omega^{k}}{\left\{\int_{C P_{n}} \kappa^{n} \Omega^{n}\right\}^{k / n}} \\
& =\frac{\int_{C P_{k}} \Omega^{k}}{\left\{\int_{C P_{*}} \Omega^{n}\right\}^{k / n}} \\
& =1 .
\end{aligned}
$$

We now let $\xi$ be a $(1,1)$-form, $\xi=\left((-1)^{1 / 2} / 2\right) \Sigma_{i, k} h_{j k} d z_{i} \wedge d \bar{z}_{k}, h_{j k}=\bar{h}_{k i}$ with de Rham decomposition

$$
\xi=\kappa \Omega+d \theta_{1}+\delta \theta_{3},
$$


where if ${ }^{*}$ denotes the Hodge star isomorphism, then 1) $\delta=-{ }^{*} d^{*}$ is the adjoint of $d$ relative to the inner product

$$
(\omega, \eta)=\int_{C P_{n}} \omega \wedge * \eta,
$$

2) $\theta_{1}, \theta_{3}$ are real 1 - and 3 -forms, respectively, $\kappa$ is a constant, and 3) the decomposition (10) is orthogonal relative to the inner product (11). Finally set

$$
\Omega_{t}=\Omega+t \xi \text {. }
$$

Then for sufficiently small $t, \Omega_{t}$ determines a Hermitian metric on $C P_{n}$. Direct calculation shows (since ${ }^{*} \Omega^{k}=\Omega^{n-k} k=1, \cdots, n$ ) that for any real $M$ homologous to $C P_{k}$ (henceforth written as $M \sim C P_{k}$ )

$$
\int_{M} \Omega_{t}^{k}=1+k t\left(\kappa+\int_{M} \delta \theta_{3} \wedge \Omega^{k-1}\right)+t^{2}(\cdots)
$$

for $k=1, \cdots, n-1$, and

$$
\int_{C P_{n}} \Omega_{t}^{n}=1+k t_{\kappa}+t^{2}(\cdots)
$$

which implies that for $\sigma_{M}(t)=\int_{M} \Omega_{t}^{k} /\left\{\int_{C P_{n}} \Omega_{t}^{n}\right\}^{k / n}$,

$$
\sigma_{M}(t)=1+k t \int_{M} \delta \theta_{3} \wedge \Omega^{k-1}+t^{2}(\cdots) .
$$

Lemma 4. Let $G=S U(n+1)$-the special unitary group on $n+1$ complex variables, acting as the group of isometries of $C P_{n}-M$ a compact $2 k$-submanifold of $C P_{n}$ and define $\beta_{M}: G \rightarrow \mathrm{R}$ by

$$
\beta_{M}(g)=\int_{g \cdot M} \delta \theta_{3} \wedge \Omega^{k-1}
$$

where $g \cdot M$ is the image of $M$ under the action of $g \varepsilon G$. Then

$$
\int_{G} \beta_{M} d \mu=0
$$

where $d \mu$ is invariant measure on $G$.

Proof. It is known that the only non-zero invariant differential forms on $C P_{n}$ are linear combinations (over the real numbers) of $1, \Omega, \Omega^{2}, \cdots, \Omega^{n}$. For $g \varepsilon G$ and any form $\omega$ on $C P_{n}$, let $g \cdot \omega$ denote the pullback of $\omega$ under the action of $g$. Then $\int_{G} g \cdot \theta_{3} d \mu$ (i.e., the integral of the form-valued function $g \cdot \theta_{3}$ on $G$ ) is an invariant 3-form on $C P_{n}$ and hence zero. Also since $g$ leaves the * operator invariant, we have $g \cdot \delta \theta_{3}=\delta g \cdot \theta_{3}$. Therefore

$$
\int_{G} \beta_{M} d \mu=\int_{G}\left\{\int_{\sigma \cdot M} \delta \theta_{3} \wedge \Omega^{k-1}\right\} d \mu
$$




$$
\begin{aligned}
& =\int_{G}\left\{\int_{M} g \cdot\left(\delta \theta_{3} \wedge \Omega^{k-1}\right)\right\} d \mu \\
& =\int_{G}\left\{\int_{M} g \cdot \delta \theta_{3} \wedge g \cdot \Omega^{k-1}\right\} d \mu \\
& =\int_{G}\left\{\int_{M} \delta\left(g \cdot \theta_{3}\right) \wedge \Omega^{k-1}\right\} d \mu \\
& =\int_{M} \delta\left\{\int_{G} g \cdot \theta_{3} d \mu\right\} \wedge \Omega^{k-1} \\
& =0
\end{aligned}
$$

which implies the lemma.

Note that since $G$ is connected, $M$ and $g \cdot M$ are homologous, for all $g \varepsilon G$. Therefore, if there exists $M_{0} \sim C P_{k}$ such that $\int_{M_{0}} \delta \theta_{3} \wedge \Omega^{k-1} \neq 0$, then

$$
a \equiv \inf _{M \sim C P_{k}} \int_{M} \delta \theta_{3} \wedge \Omega^{k-1}<0<\sup _{M \sim C P_{k}} \int_{M} \delta \theta_{3} \wedge \Omega^{k-1} \equiv b
$$

which implies by (12)

$$
\begin{aligned}
\sigma(t) & =\inf _{M \sim C P_{k}} \sigma_{M}(t) \\
& =1+\left\{\begin{array}{ll}
t a k & t>0 \\
t b k & t<0
\end{array}\right\}+t^{2}(\cdots) \\
& <1,
\end{aligned}
$$

for sufficiently small $|t|>0$. If $\int_{M} \delta \theta_{3} \wedge \Omega^{k-1}=0$ for all $M \sim C P_{k}$ then an elementary argument using Stokes Theorem implies $\delta \theta_{3}=0$, i.e., $\xi$ is Kählerian. And the theorem is proven.

We also note that for any non-Kählerian metric on $C P_{n}$ if $\lambda(\Phi)>-\infty$, then by suitable perturbation $\lambda(\Phi)$ can be increased-we look at the case $C P_{1} \subseteq C P_{2}$. Let $\Phi=\kappa \Omega+d \alpha+\delta \beta, \delta \beta \neq 0$. Then

$$
\lambda(\Phi)=\frac{\kappa+\inf _{M} \int_{M} \delta \beta}{\left\{\kappa^{2}+2 \int_{C P,} d \alpha \wedge \delta \beta\right\}^{1 / 2}}
$$

Since $d \alpha$ is of the form $d \alpha=\left[(-1)^{1 / 2} / 2\right] \bar{\partial} \partial f$ where $f: C P_{2} \rightarrow \mathbf{R}$ we can always deform $f$ to decrease the denominator, and still maintain the definiteness of the resulting Hermitian form.

The idea of using the averaging over $G$ in such a problem goes back to [12]. The direct generalization of Pu's original result, restricted to conformal deformations of Riemannian homogeneous metrics is as follows: 
Theorem 5. (P. M. Pu). Let $G$ be a compact Lie group, $H$ a closed subgroup, $G / H$ a Riemannian homogeneous space with Riemannian metric $\tau$ invariant under the action of $G, \operatorname{dim} G / H=n$. For any $k=1, \cdots, n-1$ let $\tau_{k}$ denote the $k$ dimensional volume element, $\nu_{k}$ be a homology class of $H_{k}(G / H)$, and

$$
\lambda\left(\nu_{k}\right)=\inf _{M \varepsilon \nu_{k}} \int_{M} \tau_{k} /\left\{\int_{G / H} \tau_{n}\right\}^{k / n} .
$$

Then for any $f: G / H \rightarrow \mathbf{R}^{+}-\{0\} \varepsilon C^{\infty}$, if we let $\lambda_{f}\left(\nu_{k}\right)$ denote the number corresponding to the metric $f \cdot \tau$, then

$$
\lambda\left(\nu_{k}\right) \geqq \lambda_{f}\left(\nu_{k}\right)
$$

with equality if and only if $f \equiv$ constant.

Proof. Let $h$ be the function given by

$$
h=\left\{\int_{G} g \cdot f^{k} d \mu\right\}^{1 / k} .
$$

Then by the transitivity of $G, h$ is a constant function on $G / H$. Now use Holder's Inequality and Fubini's Theorem.

\section{References}

[1] R. D. M. Accola, Differentials and extremal length on Riemann surfaces, Proc. Nat. Acad. Sci., 46 (1960) 540-543.

[2] M. Berger, Lectures on geodsics in Riemannian Geometry, Tata Institute on Fundamental Research, Bombay, 1965.

[3] - Sur les variétés d' Einstein compactes, C. R. de III Réunion du Groupement des Mathematiciens d' Expression Latine, Centre Belge de Recherches Mathemattiques, 1966.

[4] C. Blatter, Uber Extremallängen auf geschlossenen Flächen, Comment Math. Helv., 35 (1961) 153-168.

[5] W. M. Bоотнву, Some fundamental formulas for Hermitian manifolds with non-vanishing torsion, Amer. J. Math., 76 (1954) 509-534.

[6] S. S. Chern, Complex Manifolds without Potential Theory, Van Nostrand Math. Studies \#15, D. Van Nostrand Co., Princeton, 1967.

[7] - On holomorphic mappings of Hermitian manifolds of the same dimension, Proc. of Symposia in Pure Mathematics, Vol. 11, AMS, 1968, 157-170.

[8] A. Einstein, Hamiltonisches Prinzip und allgemeine Relativitäts-theorie, Preuss. Akad. Wiss. Sitz., 1916.

[9] D. Hrlbert, Die Grundlagen der physik, Nach. Geo. Wiss. Göttingen, 1915, 395.

[10] S. Kobayashi \& K. Nomizo, Foundations of Differential Geometry, Vol. II, John Wiley and Sons, New York, 1969.

[11] T. Nagano, A problem on the existence of an Einstein metric, J. Math. Soc. Japan, 19 (1967) 30-31.

[12] P. M. Pu, Some inequalities on certain non-orientable manifolds, Pacific J. Math., 11 (1952) 55-71.

[13] W. Wrrtinger, Eine Determinantenidentität und ihre Anwendung auf Analytische Gebilde in euclidischer und hermitischer Massbestimmung, Monatshefte Math. u. Physik, 44(1936) 343-365. 